EPJ Web of Conferences 24, 02001 (2012)

DOI: $10.1051 /$ epjconf/20122402001

(C) Owned by the authors, published by EDP Sciences - SIF, 2012

\title{
The geo-neutrinos
}

\author{
G. BELLINi*
}

Dipartimento di Fisica, Università di Milano and INFN, Sezione di Milano - Via Celoria 16, 20133 Milano, Italy

\begin{abstract}
The study of the antineutrinos emitted by the radioactive decays in the Earth interior is the only way to investigate how much of the terrestrial heat is produced by these decays and which is their contribution in the various Earth components. The existence of the geo-neutrinos has recently been demonstrated by Borexino and confirmed by Kamland. Kamland had found some hints already in 2005 and 2008 Nevertheless the statistics is not yet enough to discriminate among the various geological models.
\end{abstract}

\section{A few information on the neutrinos}

The neutrinos belong to one of the two families of the elementary particles, the leptons. This family includes 6 particles with the corresponding antiparticles. They are divided in three sub-families, called flavors; each of them consists of two particles and one of them is a neutrino. For this discussion on the geo-neutrinos only the first flavor is important, which includes the electron and the electron-neutrino $\nu_{e}$.

The neutrinos are particles without charge and with a very small mass, which has not been measured yet because the needed sensitivity has not been reached until now. Their probability to interact with the material is very weak. Its cross section, the square of which is proportional to the interaction probability, is fourteen orders of magnitude lower than the proton cross section and twelve with respect the electron one. This means that

*E-mail: gianpaolo.bellini@mi.infn.it

This is an Open Access article distributed under the terms of the Creative Commons Attribution License 2.0, which permits unrestricted use, distribution, and reproduction in any medium, provided the original work is properly cited. 
the neutrinos can cross also the Universe without being disturbed. This is of course true also for the neutrinos escaping from the Sun and the Earth interior. The neutrino is thus an incredible probe to study phenomena within these big systems.

The neutrinos maintain their origin: the neutrino flavor during their interactions and travel is supposed to be conserved, except for the mechanism, discovered about ten years ago, of the oscillation. Following this mechanism a neutrino can change its flavor crossing matter or vacuum. For instance an electron-neutrino can be changed to muon-neutrino with a certain probability, which depends on the neutrino energy, on the matter composition and other specific parameters. In the experimental study of the neutrinos the oscillation phenomenon has to be taken into account.

But which is the role of the neutrinos in the structure of the matter? We know that the matter includes three types of particles: protons, neutrons, electrons. The protons and the electrons are stable, free neutrons have a lifetime of $885.7 \mathrm{~s}$. The neutrinos are stable and they are uncoupled from the matter; they are produced in the decay of unstable particles. In fact, in addition to the particles mentioned above, there are a few hundreds of unstable particles, with a very short lifetime (from $10^{-8}$ to $10^{-27} \mathrm{~s}$ ). They decay (are transformed) into other particles, which can be unstable or stable: the unstable ones also decay and finally only the stable particles remain (we consider the neutron as stable in this context).

In the Big Bang all the elementary particles have been produced, unstable ones included. They decayed onto neutrons, protons and electrons, which matched into atoms, and the neutrinos which, staying uncoupled, remained in the Universe. To our knowledge 300 relic neutrinos per square meter are present in the whole Universe.

\section{A sketch of the Earth matter}

The Earth is made up by various layers. Going from the Earth center to its surface we find:

- an inner Core, solid due to the high pressure ( 330 GPa), despite the high temperature $(\sim 5700 \mathrm{~K})$ : its size is approximately that of the Moon and it consists essentially of a Fe-Ni alloy.

- an outer Core, $2260 \mathrm{~km}$ thick, liquid (4100-5800 K of temperature), it also consists of a Fe-Ni alloy with a $10 \%$ of light elements (S,O); the motion of this conductive liquid produces the geo-dynamo. 
- a Mantle-Core transition, $200 \mathrm{~km}$ thick.

- a lower Mantle, $\sim 2000 \mathrm{~km}$ thick, with an high $\mathrm{Mg} / \mathrm{Fe}$ ratio, at high temperature (600-3700 K) and high pressure, viscose; due to the non homogeneous distribution of the caloric energy in it, thermal convections are produced which are connected to volcanic activities and to movements of the tectonic plates.

- a transition region, 400-650 km thick, which has seismic discontinuities; partial melting in it is the source of the mid-ocean ridges basalts, important because mantle material emerges in them.

- an upper Mantle, more than $350 \mathrm{~km}$ thick, composed by peridotite type rocks, which includes the highly viscose so called astenosphere, on which the litospheric tectonic plates are floating.

- the Crust, which can be divided in Oceanic Crust, $\sim 10 \mathrm{~km}$ thick, which includes the mid-oceanic ridges, and the Continental Crust, 30$70 \mathrm{~km}$ thick, igneous, metamorphic, with sedimentary rocks.

The methods used by the geologists can be classified in geophysics and geochemistry categories. The physics methods are based upon the study of the diffusion through the Earth of the seismic waves: the transverse (shear) waves do not propagate in the liquid matter and there is a direct relation between the waves velocity and the density of the crossed material. But the physics methods do not provide any information on the Earth composition, which on the other hands is studied by the geochemists.

The geochemistry uses two approaches: the analysis of the direct rock samples, and models. It is possible to obtain crust samples by means of bore-holes (the deepest one has $12 \mathrm{~km}$ ), while mantle investigations can be done using the upper mantle peridotites in the mid ocean ridges and the rocks brought up by different types of volcanism.

The geochemical models assume that the chemical composition of the solar system has to be the same for all its components; the composition of the chondritic meteorites and the Sun composition are so assumed as a model for the Earth.

The reference model for the geologists is the Bulk Silicate Earth (BSE), which studies the evolution of the Earth starting before the crust differentiation and after the Fe-Ni core separation; but in this model also data provided by the geochemical models are taken into account. 


\section{The Earth heat flow}

The geological estimation of the heat within the Earth shows a large range. The evaluation based upon the conductive heath flow from the bore-hole temperature gradient gives $31 \pm 1 \mathrm{TW}$, while the study of the gradient $v s$. distance from the mid-ocean ridges (taking into account also the effects of the water cooling) gives $46 \pm 1 \mathrm{TW}$.

The previsions of how much of this heat is due to the radioactive decays are also different: in the BSE model only $19 \mathrm{TW}$ are foreseen for the crust and the uppermost portion of the mantle, but also for this model this evaluation can change if we assume an homogeneous distribution in the mantle or a radiogenic accumulation at the bottom of it. Other hypotheses concern a possible ${ }^{40} \mathrm{~K}$ presence in the core and an accumulation of Uranium around the core, which would produce a reactor (geo-reactor) of 3-6 TW of power. In this case the heat from radiogenic source could reach also $44 \mathrm{TW}$.

The radioactive decays in the Earth are due to the ${ }^{238} \mathrm{U}$ and ${ }^{232} \mathrm{Th}$ families and to ${ }^{40} \mathrm{~K}$. All these decays are producing heat:

$$
\begin{aligned}
& { }^{238} \mathrm{U} \rightarrow{ }^{206} \mathrm{~Pb}+8 \alpha+8 e^{-}+6 \text { anti-neutrinos }+51.7 \mathrm{MeV} \\
& { }^{232} \mathrm{Th} \rightarrow{ }^{208} \mathrm{~Pb}+6 \alpha+4 e^{-}+4 \text { anti-neutrinos }+42.8 \mathrm{MeV} \\
& { }^{40} \mathrm{~K} \rightarrow{ }^{40} \mathrm{Ca}+e^{-}+1 \text { anti-neutrino }+1.32 \mathrm{MeV}
\end{aligned}
$$

Their energy spectra are shown in fig. 1.

The geo-neutrino events, in a detector liquid scintillator based, are well tagged.

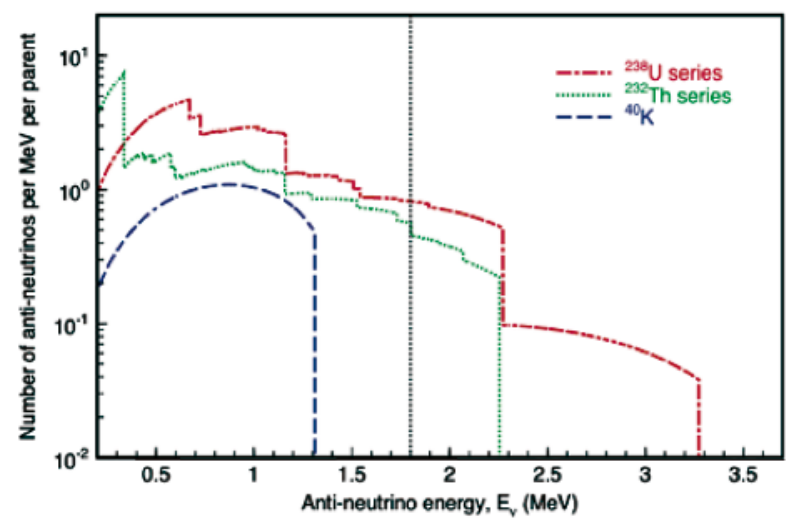

Fig. 1 


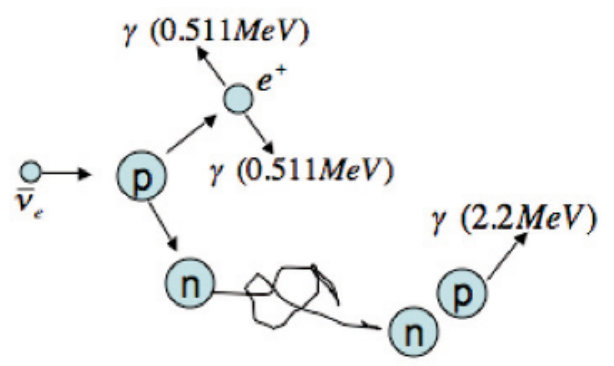

Fig. 2

In the collision of the $\bar{\nu}_{e}$ on proton (see fig. 2), one positron (the antiparticle of the electron) is emitted together with a neutron. The positron annihilates immediately meeting an electron with the emission of two prompt $\gamma \mathrm{s}$ of $0.511 \mathrm{MeV}$ each. The neutron thermalizes with a delay of $\sim 260 \mu \mathrm{s}$ and is captured by a proton, producing a photon of $2.2 \mathrm{MeV}$ of energy. The tagging consists of two $0.511 \mathrm{MeV} \gamma \mathrm{s}$, followed by one $2.2 \mathrm{MeV} \gamma$ with a delay of about $260 \mu \mathrm{s}$.

This strong tagging is very important in order to disentangle the $\bar{\nu}_{e} \mathrm{~s}$ events from the background.

The $\bar{\nu}_{e}$ interactions described above have a lower threshold at $1.8 \mathrm{MeV}$ (vertical line in fig. 1); the consequence is that the possible geo-neutrinos from ${ }^{40} \mathrm{~K}$ cannot be detected, while the ones emitted in the decays of the ${ }^{238} \mathrm{U}$ and ${ }^{232} \mathrm{Th}$ families can be studied over $1.8 \mathrm{MeV}$.

\section{The detection of the geo-neutrinos}

Presently only two experiments in the world are able to study the Geoneutrinos: Borexino at the underground Gran Sasso laboratory in Italy $[1,2]$ and Kamland at the underground Kamioka laboratory in Japan [3]. Both are using liquid scintillator (300 tons in Borexino and 1 kton in Kamland), are shielded with respect to the cosmic rays by the rock overburden and have a similar structure, with the difference that Borexino has a much higher radiopurity.

In fig. 3 a sketch of Borexino is shown.

The nylon vessel, $125 \mu \mathrm{m}$ thick, $8.5 \mathrm{~m}$ of diameter, contains 270 tons of two components liquid scintillator: Pseudocumene (PC) plus a fluor (PPO). It is surrounded by $1350 \mathrm{~m}^{3}$ of pure PC ( $\sim 2 \mathrm{~m}$ of buffer in all directions) within a stainless sphere of $13.7 \mathrm{~m}$ diameter. Between the sphere and the 


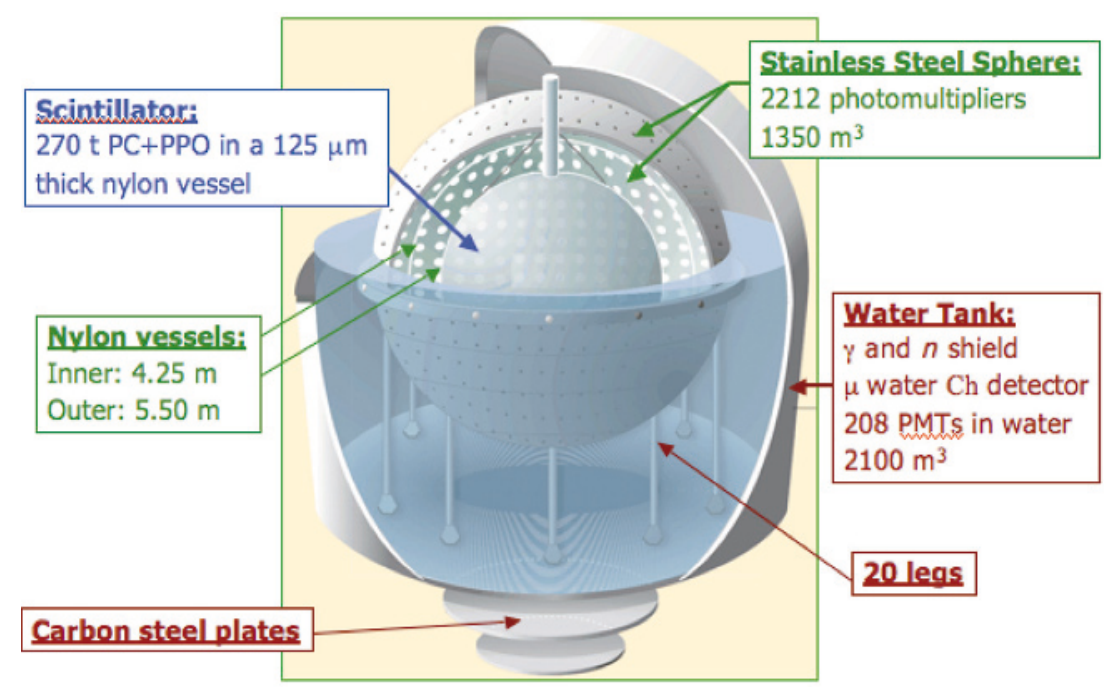

Fig. 3

nylon vessel a second nylon balloon serves as barrier against the radon emission by the stainless steel and by the 2212 photomultipliers supported by the sphere. Finally a water tank, containing $2100 \mathrm{~m}^{3}$ of highly purified water surrounds the sphere, assuring a further shielding of at least $2 \mathrm{~m}$ in all directions.

The basic idea followed in this design is to assure a good shielding, first (water) from the external radiations of the rocks and the environment, second (buffer PC) from the radiations survived through the water, and from the photomultiplier and stainless steel emission. The very thin nylon layer (made of material chosen with high radiopurity and extruded in a clean room) of the Inner Vessel keeps at very low level its emission, but requires a buoyancy close to zero, which is assured by the buffer liquid having an almost identical density as the scintillator. The photomultipliers are coupled with optical concentrators, which turn towards the photocatodes the photons produced by the interactions in the scintillator, thus assuring a $30 \%$ optical coverage.

At the Gran Sasso underground laboratory $\sim 1.1 \frac{\mathrm{muons}}{\mathrm{m}^{2} \cdot h}$ cross the overburden of about $2700 \mathrm{~m}$ of rocks. These muons can produce radioactive nuclides and neutrons, and it is very important to detect them. As a consequence a muon veto has been installed in the external water tank, with 208 photomultipliers, exploiting the Cherenkov light produced by the muons in the water. 
Table I

\begin{tabular}{|c|c|c|c|}
\hline & Material & $\begin{array}{l}\text { Typical conc. of } \\
\text { the unpurified } \\
\text { materials }\end{array}$ & $\begin{array}{l}\text { Radiopurity levels } \\
\text { in the } \mathrm{Bx}\end{array}$ \\
\hline${ }^{14} \mathrm{C}$ & scintillator & ${ }^{14} \mathrm{C} /{ }^{12} \mathrm{C}<10^{-12}$ & ${ }^{14} \mathrm{C} /{ }^{12} \mathrm{C} \approx 2 \cdot 10^{-18}$ \\
\hline${ }^{238} \mathrm{U},{ }^{232} \mathrm{Th}$ equiv. & $\begin{array}{l}\text {-Hall C dust } \\
\text {-stainless steel } \\
\text {-nylon }\end{array}$ & $\begin{array}{l}\sim 1 \mathrm{ppm} \\
\sim 1 \mathrm{ppb} \\
\sim 1 \mathrm{ppt}\end{array}$ & $10^{-17}-10^{-18} \mathrm{~g} / \mathrm{g}$ \\
\hline $\mathrm{K}$ & Hall C dust & $\sim 1 \mathrm{ppm}$ & $10^{-14} \mathrm{~g} / \mathrm{g}$ \\
\hline${ }^{222} \mathrm{Rn}$ & $\begin{array}{l}\text {-external air } \\
\text {-air underground }\end{array}$ & $\begin{array}{l}\sim 20 \mathrm{~Bq} / \mathrm{m}^{3} \\
\sim 40-100 \mathrm{~Bq} / \mathrm{m}^{3}\end{array}$ & $<1 \mu \mathrm{Bq} / \mathrm{m}^{3}$ \\
\hline $\begin{array}{l}{ }^{85} \mathrm{Kr} \\
{ }^{39} \mathrm{Ar}\end{array}$ & in $\mathrm{N}_{2}$ for stripping & $\begin{array}{l}\sim 1.1 \mathrm{~Bq} / \mathrm{m}^{3} \\
\sim 13 \mathrm{mBq} / \mathrm{m}^{3}\end{array}$ & $\begin{array}{l}\sim 0.16 \mathrm{mBq} / \mathrm{m}^{3} \\
\sim 0.5 \mathrm{mBq} / \mathrm{m}^{3}\end{array}$ \\
\hline $\begin{array}{l}-{ }^{222} \mathrm{Rn} \\
-238 \mathrm{U},{ }^{232} \mathrm{Th} \text { equiv. }\end{array}$ & LNGS - Hall C water & $\begin{array}{l}\sim 50 \mathrm{~Bq} / \mathrm{m}^{3} \\
\sim 10^{-10} \mathrm{~g} / \mathrm{g}\end{array}$ & $\begin{array}{l}\text { Water } \\
\begin{array}{l}\sim 30 \mu \mathrm{Bq} / \mathrm{m}^{3} \\
\sim 10^{-14} \mathrm{~g} / \mathrm{g}\end{array}\end{array}$ \\
\hline
\end{tabular}

All materials are selected to keep their radioactive level as low as possible; the water, the $\mathrm{PC}$ and the scintillator have been radio-purified using techniques developed on purpose and are sparged with $\mathrm{N}_{2}$, also highly purified from ${ }^{222} \mathrm{Rn},{ }^{39} \mathrm{Ar},{ }^{85} \mathrm{Kr}$. The result is that the Borexino detector reaches an unprecedented very high radio-purity (see table I); in this aspect it is a unique detector.

In table I the contaminants of various materials used in the Borexino detector or in the radio-purification processes are shown: for the regular unpurified materials, and in Borexino, after the purification.

\section{The background}

The geo-neutrino interactions are rare and it is very important to keep under control the background. The main sources of it are the $\bar{\nu}_{e S}$ emitted by the nuclear reactors, the radioactivity of the detector components and the events induced by cosmic muons crossing the overburden and the accidental coincidences. 
Table II

\begin{tabular}{|c|c|c|}
\hline Source & Borexino [events/(100 ton-yr)] & KamLAND [4126 ton-yr] \\
\hline Cosmogenic ${ }^{9} \mathrm{Li}$ and ${ }^{3} \mathrm{He}$ & $0.03 \pm 0.02$ & $2 \pm 0.1\left({ }^{9} \mathrm{Li}\right)$ \\
\hline $\begin{array}{l}\text { Fast neutrons from } \mu \text { in Water } \\
\text { Tank }\end{array}$ & $<0.01$ (measured) & \multirow[t]{2}{*}{$<2.8$} \\
\hline Fast neutrons from $\mu$ in rock & $<0.04(\mathrm{MC})$ & \\
\hline Non-identified muons & $0.011 \pm 0.001$ & \\
\hline Accidental coincidences & $0.080 \pm 0.001$ & $77.4 \pm 0.1$ \\
\hline Time correlated background & $<0.026$ & \\
\hline$(\gamma, \mathrm{n})$ reactions & $<0.003$ & \\
\hline Spontaneous fission in PMTs & $0.003 \pm 0.0003$ & \\
\hline $\begin{array}{l}(\alpha, \mathrm{n}) \text { reaction in the } \\
\text { scintillator }\left[{ }^{210} \mathrm{Po}\right]\end{array}$ & $0.014 \pm 0.001$ & $165.3 \pm 18.2$ \\
\hline $\begin{array}{l}(\alpha, \mathrm{n}) \text { reaction in the buffer } \\
{\left[{ }^{210} \mathrm{Po}\right]}\end{array}$ & $<0.061$ & \\
\hline TOTAL & $0.14 \pm 0.02$ & \\
\hline
\end{tabular}

In both the experiments, Borexino and Kamland, the reactor $\bar{\nu}_{e}$ flux has been carefully evaluated taking into account: the various reactor powers and their possible changes month per month during data taking, the fuel composition, the distances reactors-detector, the effects of the neutrino oscillation (an $\bar{\nu}_{e}$ has a certain probability to oscillate in to a $\bar{\nu}_{\mu}$ ). The expression used for this evaluation is

$$
\Phi\left(E_{\bar{v}_{e}}\right)=\sum_{r=1}^{N_{\text {react }}} \sum_{m=1}^{N_{\text {month }}} \frac{T_{m}}{4 \pi L_{r}^{2}} P_{r m} \sum_{i=1}^{4} \frac{f_{r i}}{E_{i}} \Phi_{i}\left(E_{\bar{\nu}_{e}}\right) P_{e e}\left(E_{\bar{\nu}_{e}} ; \vartheta_{12}, \Delta m_{21}^{2}, L_{r}\right),
$$

where $P_{r m}$ is the power per each reactor and month, obtained from the data base and the documents of the IAEA and EDF; $f_{r i}$ is the power fraction of isotopes: ${ }^{235} \mathrm{U},{ }^{238} \mathrm{U},{ }^{239} \mathrm{Pu},{ }^{241} \mathrm{Pu} ; L_{r}$, the distance reactor-detector; $T_{m}$, the data taking of the detector in months; $P_{e e}$, the survival probability of the $\bar{\nu}_{e}$ due to the oscillation phenomenon; $\Phi_{i}\left(E_{\bar{\nu}_{e}}\right)$ the antineutrino flux from each reactor. The three sum operators $\Sigma$ refer to the loops over reactors $(r)$, over months $(m)$ and over isotopes $(i)$, respectively.

The systematic errors of this calculation come mostly from the fuel composition, $\Phi_{i}\left(E_{\bar{\nu}_{e}}\right)$, the reactor power per month, the oscillation parameters. A further contribution is due to the long-lived isotopes of the nuclear radioactive wastes. 
In the case of the estimation at the Gran Sasso site of the total flux from reactor $\bar{\nu}_{e} \mathrm{~s}$, the total systematic errors introduce in the flux an uncertainty of $5.38 \%$. For this flux calculation, 194 (Europe) and 245 (rest of the world) nuclear plants have been considered: of course the contribution is mostly due to the Europe reactors.

In the Kamioka site the background due to the reactor $\bar{\nu}_{e}$ is much more important than in the Gran Sasso case: the flux is more than 7 times higher, due to the big number of nuclear plants in Japan.

The second contribution to the background is due to the radioactivity in the detector itself and in the environments. $(\alpha, n)$ and $(\gamma, n)$ interactions can mimic $\bar{\nu}$ events. This background is almost negligible in Borexino, due to the very high radiopurity, but this is not the case of Kamland because its radio-contaminants are a few orders of magnitude higher than in Borexino (see table II).

A further background is due to the events produced by the residual cosmic muons crossing the overburden. They can produce either $(\beta-n)$ emitter nuclides or neutrons, which reach the scintillator, just giving a prompt and a delay signal. These fake events can be neutralized with proper time vetoes after the detected muons.

Finally also the accidental coincidences, coupled to some signal in the scintillator, may mimic good events. In Borexino the accidentals in the geo-neutrino energy range are very small. This is not the case of Kamland, where due to the radioactive contaminants, the rate is high (see table II).

\section{The results achieved until now}

The data set analyzed until now by Borexino has been collected during 537.2 days of data taking with a fiducial exposure (which takes into account the analysis cuts) of 252.6 ton-year [4].

In fig. 4 the expected energy spectrum of the prompt positrons, simulated in the Borexino detector, is shown. The white area corresponds to the events produced by the reactor $\bar{\nu}_{e}$ events, while the shaded area are the positrons from the geo-neutrino interactions (the red line is the same spectrum, once subtracted the reactor $\bar{\nu}_{e}$ events)

In fig. 5 the measured data is shown. The black points are the experimental results, while the plots are the result of the fit carried out via an unbinned Maximum Likelihood function. Again the shaded area corresponds to the geoneutrino events, the white to the events from rector $\bar{\nu}_{e}$, while the blue area is the background [pay attention that the energy scale in the plots 


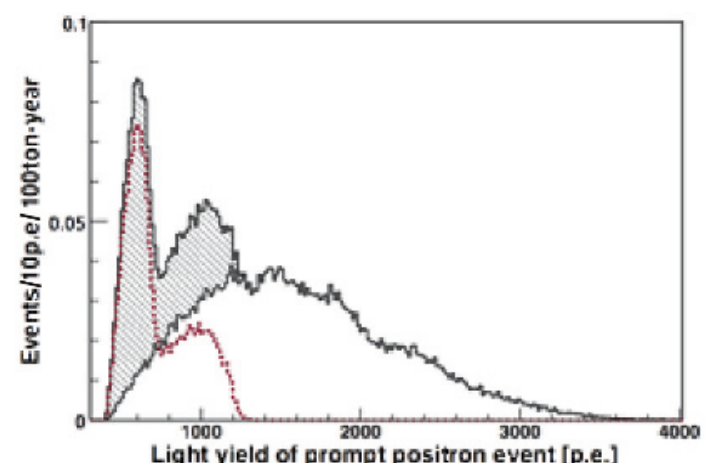

Fig. 4

of figs. 4 and 5 are in photoelectrons].

In fig. 6 the best fit parameters from the likelihood analysis is shown together with a comparison with the geological models. The hypothesis of no evidence of geoneutrinos is rejected with $99.997 \%$ Confidence Level, corresponding to 4.2 sigma. The statistics is not yet enough to discriminate among the BSE, the maximum radiogenic model (the whole Earth heat is due to the radioactive decays) and the minimum radiogenic model (the radioactive decays account only of the heat present in the Earth Crust). Nevertheless the maximum radiogenic model seems slightly preferred.

On the other hand the Borexino data allow to rule out the model based upon the existence of a 3-6 TW geo-reactor.

The signal/noise ratio in the Borexino data is $2.5 / 1$; in the first bin of fig. 5 , the signal/noise ratio is $\approx 18 / 1$.

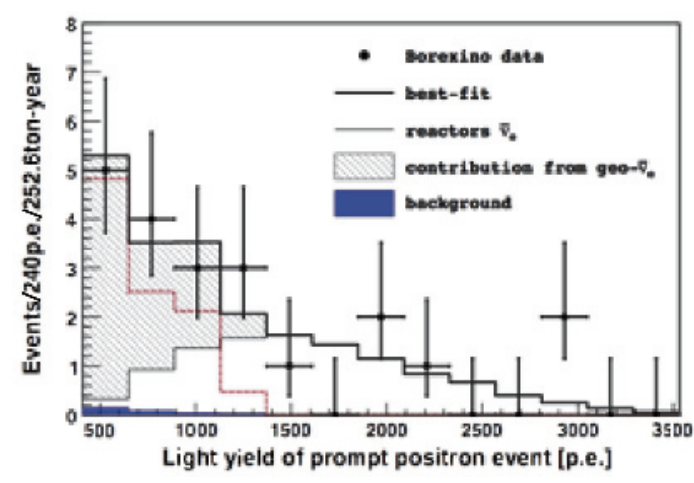

Fig. 5 


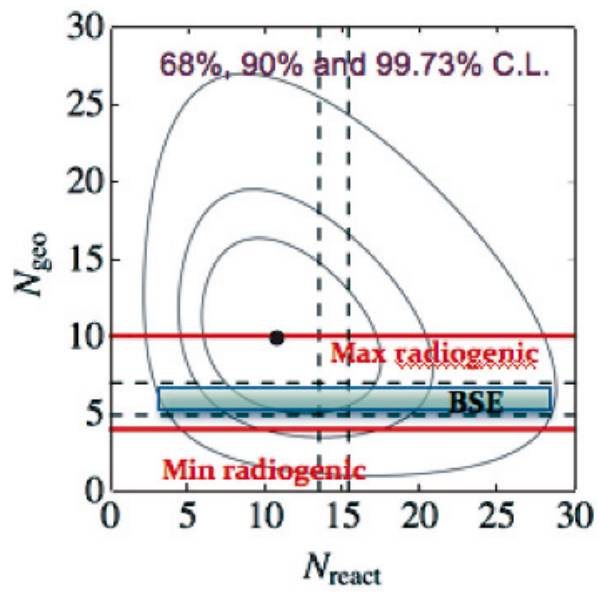

Fig. 6

The Kamland data set has been taken during 2135 days, with a fiducial exposure of 4126 ton-year [5]. In the bottom of fig. 7 the Kamland results are shown: the black points are the experimental data, while the colored areas give the various contributions: the white area corresponds to the events due to the reactor $\bar{\nu}_{e S}$, the red area to the accidental coincidences, the green area to the fake events produced by the $\alpha_{\mathrm{S}}$ radio-produced. Finally the blue

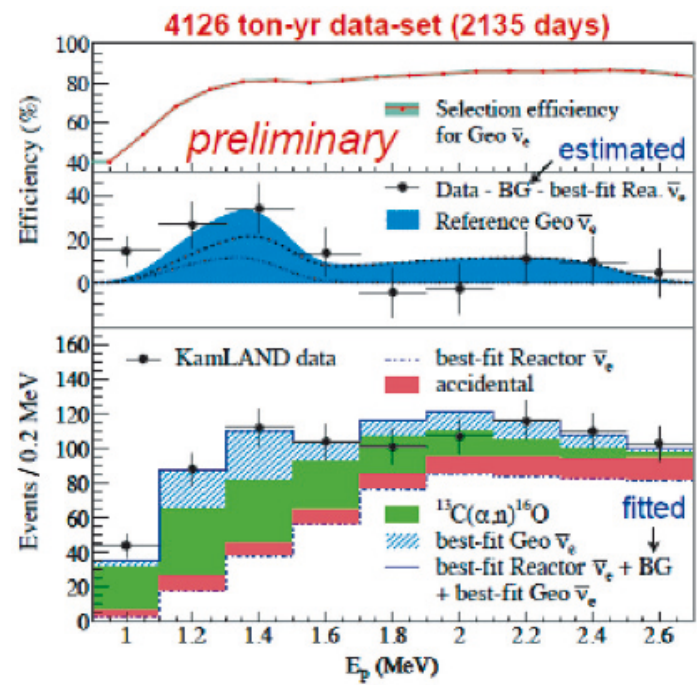

Fig. 7 
dashed area represents the geo-neutrinos.

In the intermediate plot only the fitted geo-neutrino distribution is shown, while on the top the detector efficiency in reconstructing geoneutrinos is reproduced. The Kamland data slightly favor the BSE model, even if, as the Borexino data, does not reach evidence for one of the geological models. The null geo-neutrino signal is excluded at $99.55 \%$ Confidence Level.

In Kamland the signal/noise ratio is $1 / 15$.

Hints of geo-neutrino detection have been published by Kamland already in 2005 [6] and 2008 [7], this last with a Confidence Level at 2.5 sigma.

\section{Conclusions}

The evidence of the geo-neutrinos is now very robust. Nevertheless the limited statistics does not allow a discrimination among the different models, with the exception of the geo-reactor model, which is ruled out by the Borexino results.

Borexino plans to have enough statistics, by the end 2011, to discriminate among the various geological models. By the end 2013 Borexino will be probably able to measure the $\mathrm{Th} / \mathrm{U}$ ratio.

\section{References}

[1] Alimonti G. et al. (BOREXINO Collaboration), Nucl. Instrum. Methods $A, 600$ (2009) 568.

[2] Alimonti G. et al. (BOREXINO Collaboration), Nucl. Instrum. Methods A, 609 (2009) 58.

[3] Eguchi K. et al. (KAMLAND Collaboration), Phys. Rev. Lett., 90 (2003) 021802.

[4] Bellini G. et al. (BOREXINO Collaboration), Phys. Lett. B, 687 (2010) 299.

[5] Shimizu I.,Talk presented at "Neutrino Geoscience 2010", $7^{\text {th }}$ October, 2010.

[6] Araki T. et al. (KAMLAND Collaboration), Nature, 436 (2005) 499.

[7] Abe S. et al. (KAMLAND Collaboration), Phys. Rev. Lett., 100 (2008) 221803. 